\title{
الفكر الغربي: مشروع رؤية نقدية
}

\section{عبد الوهاب المسيري}

يمكن أن نقرر ابتداءاً أن العقل العربي الإسلامي في العصر الحديث قد دخل -نتيجة لظروف عديدة- حلقة مفرغة ستزيد من تفككه وتاكله وتقويض ثقته بنفسه وضمور إبداعه. فالنهضة أصبحت تعنى -بالنسبة للكثيرين- اللحاق بالآخر (الغرب) والابتعاد عن الذات والتراث، بل وإعادة صياغتهما مما يتفق مع المقاييس الغربية. والجها المعريف الوحيد للمشروع هو نقل ما يقوله الآخر إما دون اجتهاد أو بقليل من التعديل والتحوير. وهذا يؤدي إلى مزيد من الاعتماد المعرفي على الآخر والتبعية المعرفية له والركض وراءه.

ولذا لا غرو أن الإنسان العربي المسلم لم يأت بمعرفة جديدة في العصر الحديث، ولم يؤسس علوماً ذات بال، ولم يسهم في إثراء الفكر الإنساني. ولا يمكن للإنسان المسلم أن يبدع ويولد معارف حديثة من تراثه ونماذجه المعرفية إلا بعد أن يتحرر من قبضة الغرب عن طريق ما نسميه بالمحور النقدي للمشروع الحضاري الإسلامي. وأعتقد أن كل من يود أن يطور مشروعاً معرفياً حضارياً مستقلاً عليه أن يبدأ -شاء أم أبى - بنقد المشروع الغربي نظراً لذيوعه وهيمنته.

ويمكن لمذه العملية النقدية أن تنجز ما يلي:

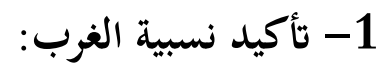

إذا كان الغرب قد تحول إلى مطلق فيجب أن يستعيد نسبيته وتاريخيته وزمنيته، وإذا كان يشغل المركز، فيجب أن يصبح مرة أخرى عنصراً واحداً ضمن عناصر أخرى تُكوّن عالمُ الإنسان، وإذا كان يعتبر نفسه عالمياً

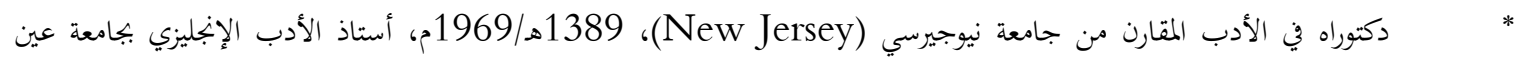
شمس بالقاهرة. 
وعاماً فيجب أن نبين خصوصيته ومحلته... أي: أن الغرب يجب أن يصبح "غربياً" مرة أخرى لا "عالمياً"، وهذا لا يمكن أن يتم إلا باستعادة المنظور العلمي المقارن، بحيث يصبح التشكيل الحضاري الغربي تشكيلاً حضارياً واحداً له خصوصيته ومسماته، تماماً مثلما لكل التشكيلات الأخرى خصوصيتها وسماتها.

والمنظور المقارن لا يعني علاقة التأثير والتأثر الشائعة في الدراسات الجامعية، وإنما يعني محاولة الوصول إلى رؤية عالمية حق من خلال مقارنة البنى والأشكال الحضارية المختلفة التي تَعَمَل الإنسان من خلالها مع العالمها

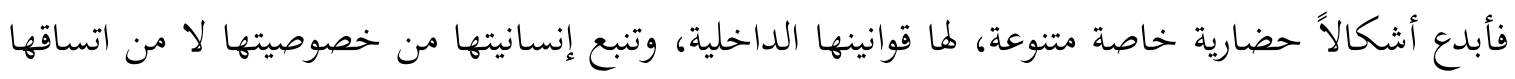
مع قانون عالمي عام وهمي هو في غاية الأمر "قانون غربي".

2- تراجع المركزية الغربية:

ومما يساعد على هذا الأمر أن الحضارة الغربية قد فقدت مركزيتها المزعومة حتى على المستوى المادي، إذ ظهرت مراكز حضارية أخرى ناجحة حتى بالمعايير المادية المقبولة لدى الحضارة العلمانية (مثل اليابان والصين وشرق آسيا). وعملية تحول الغرب من مركز مطلق إلى أحد التككيلات الحضارية بتعل من الممكن لنا أن

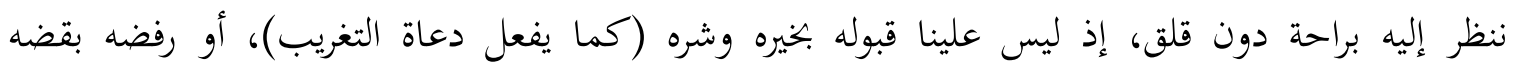
وقضيضه (كما يفعل بعض السلفيين)، وإغما يككنا دراسته كمتتالية حضارية تتسم بما تتسم به من سلبيات وإيابابيات.

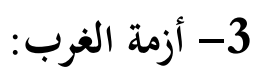

وإذا كان الغرب قد حقق إطلاقه ومركزيته من خلال الانتصارات المعرفية والمادية التي حققها في المراحل الأولى من ظهور النموذج العقلاني المادي، فقد حان الوقت لأن نعيد النظر في هذه الانتصارات والنجاحات، ونبين نقط القصور التي ظهرت من خلال التطبيقات المختلفة لنموذجه المعريف، خاصة ابتداءً من منتصف القرن

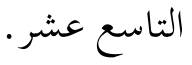

ومن المفارقات التي تستحق التسجيل أن دعاة التغريب واللحاق بالغرب لا يزالون يدورون في إطار عقلانية القرن الثامن عشر وعلوم القرن التاسع عشر، ويكررون تفاؤل الغرب بخصوص مستقبله، في الوقت الذي 
سقطت فيه عقلانية القرن الثامن عشر، وظهر مدى قصورها، وتآكلت السبية البسيطة التي تسند إليها علوم القرن التاسع عشر، وتخلّى كثير من المفكرين الغربيين عن تفاؤهم بخصوص حضارقم التي لم تعد تشعر بالثقة الكاملة بنفسها كما كانت تفعل حتى فاية القرن التاسع عشر، وفقدت كثيراً من إحساسها بمكانتها الخاصة في التاريخ ومركزيتها وعالميتها.

وهذا أمر طبيعي ومتوقع مع تصاعد أزمات هذه الحضارة ابتداءًا من حربيها العالميتين (أي الغربيتين)، وانتهاءاً بمشاكلها المتنوعة الكثيرة، مثل: تآكل مؤسسة الأسرة، وانتشار (الإيدز) والمخدرات، وتزايد اغتراب الإنسان

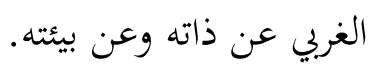

\section{أولاً: الفكر الغربي الاحتجاجي أو المضاد}

يعود تاريخ الفكر الاحتجاجي إلى عصر النهضة في الغرب ذاته، أي مع ظهور النموذج العقلاني المادي، إلا أنه ظل متوارياً بسبب انتصارات الحضارة الغربية المادية والمعنوية المذهلة. ولكن مع دخول هذه الحضارة مرحلة الأزمة بدأ هذا الفكر الاحتجاجي يكتسب مزيداً من المركزية والمصداقية. وقد حاول هذا الفكر وصف الأزمة والتعبير عنها بل وتحاوزها. ولا بد من الاستفادة من هذا الفكر وأدبياته في محاولة استرداد المنظور النقدي بتاه الحضارة الغربية.

ولا بد أن نوفر للقارئ العربي أهم أدبيات هذا الفكر الذي يشمل كل فروع المعرفة: النقد الأدبي، واللغة، والفلسفة، وعلم البيئة، والعلوم الطبيعة، والتاريخ... الخُ، وسنجد أن النموذج الكامن وراء هذا الفكر ينطوي على قدر من الرفض للنماذج العلمية الكمية العامة التي تمل الكيف وما وراء الطبيعة والمطلق والخصوصية. ووجود هذه الأدبيات بالعربية سيجعل الحوار بخصوص المشروع الغربي أكثر ترتيباً وعمقاً.

فعلى سبيل المثال أثناء الاحتفال المئوي للثورة الفرنسية انبرى دعاة التغريب للتبشير المتكرر مثثاليات الثورة الفرنسية مثل: الحرية والإخاء والمساواة، وللحديث عن "دَيْنا" للثورة الفرنسية، وعن إعلان حقوق الإنسان وغيرها من الموضوعات المستهلكة التي لم تعد بتح من يدافع عنها في الغرب. بينما نجد أن الاحتفال بالعيد 
المئوي الثاني في فرنسا ذاتا كان يتسم بالتوتر الشديد والانقسام، فلو توافرت بعض النصوص "المراجِعة" بخصوص الثورة الفرنسية لارتفع مستوى الحوار.

ولذا نتترح توفير بجموعة من النصوص والأبحاث "لمراجعة" و "الاحتجاجية" في الموضوعات التالية (ويكننا الاستعانة ببعض الباحثين الأجانب الذين هم أكثر إلماماً منا بطبيعة الفكر الغربي الاحتجاجي وأدبياته نظراً لمتابعتهم ومعايشتهم له): (n) أ- الحداثة:

ظهرت في الغرب دراسات كثيرة للغاية عن انتهاء الحداثة، وفشلها وعدميتها، ولا إنسانيتها، وضرورة بحاوزها. وهناك دوريات متخصصة في نقد الحداثة، كما أن هناك دوريات فلسفية وأدبية ذات توجه ديني (مسيحي) تنشر دراسات نقدية معمقة في هذا الموضوع. وهناك نقد للحداثة داخل الفكر الماركسي في غاية الأهمية (لوكاش، وجراف، وجيمسون، وإيجلتون).

ب- نظريات التنمية (والتقدم):

ظهرت دراسات عديدة عن مفهوم التنمية تبيّن أنه مفهوم كَمِي لا يتعامل مع الكيف، ومادي لا يتعامل مع الإنسان، وعام لا يتعامل مع الخاص والفردي، أي: أنه قاصر عن الإحاطة بكثير من جوانب الظاهرة الإنسانية. ومع تصاعد أزمة البيئة بدأ الكثيرون يتحدثون عن حدود التقدم والتنمية. كما أن فشل معظم التجارب التنموية في العالم الثالث التي تستند إلى مفاهيم التنمية الغربية بدأ هو الآخر ينتج أدبيات تطالب بفتح باب الاجتهاد بخصوص هذا المفهوم الموري للتشكيل الحضاري الغربي. (ولفجانج ساكس: قاموس التنمية).

\section{ج- فكر أحزاب الحضر والمدافعين عن البيئة:}

وهو من أهم أنواع التفكير المضاد، فإذا كان الفكر الغربي العلماني مبيناً على التحكم في الأرض وغزوها، فهذا فكر يصدر عن فكرة التواؤم معها، ومن ثم فهو في أعماقه -ومن الناحية المعرفية- فكر ديني، إذ 
يفترض أن ثمة حدوداً وقيوداً عل الإرادة الإنسانية، وأن الإنسان ليس مجرد ظاهرة طبيعة، وإنما هو على

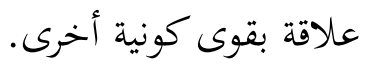

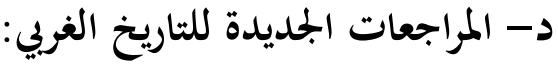

ظهرت دراسات تاريخية عديدة للتاريخ الغربي والفكر تعيد النظر فيه وفي مسلماته، فأعيد كتابة تاريخ

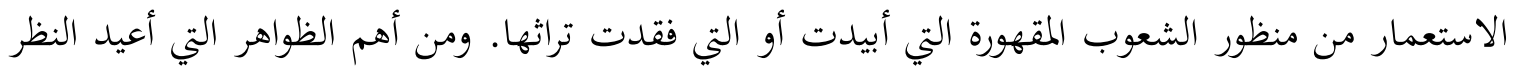
فيها ظاهرة الثورة الفرنسية، فقد ظهر ما يقرب من خمسمائة كتاب عن الثورة الفرنسية، أساساً باللغتين الإنجليزية والفرنسية، وبعض هذه الكتب يطرح رؤية جديدة تماماً.

فمثلاً تبين إحدى الدراسات أن العنف الذي صاحب الثورة لم يكن بجرد ظاهرة عرضية، وإنما كان سمة بنيوية. فالعنف -حسب ما تطرحه هذه الدراسة- كان هو الوسيلة الوحيدة المتاحة للدولة القومية (التي

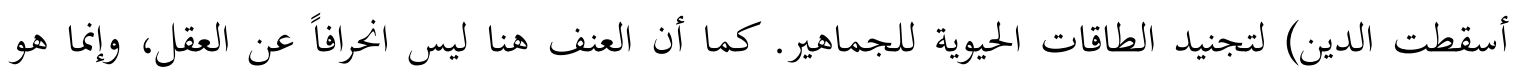
إعادة صياغة الواقع بما يتفق مع مقاييس العقل الرياضية الباردة غير الإنسانية. وتشير دراسات أخرى إلى موقف الثورة الفرنسية من الأقليات، وكيف أخها أبادقا، إما فعلياً أو بحازياً عن طريق علمنتها وإسقاط سمامًا الخصوصية.

وهناك دراسة ثالثة تبين أن الثورة أدت إلى إعاقة تطور فرنسا الاقتصادي، ومن ثم أعطت الفرصة لإنجلترا لتصبح الدولة الصناعية العظمى دون منازع، وهناك دراسات عن تزايد معدلات الطلاق بعد الثورة وعدد الأطفال غير الشرعيين وغيرها من الموضوعات الاحتجاجية. وهناك معجم نقدي للثورة الفرنسية.

\section{هـ - المراجعات الجديدة في علم النفس وعلم اللغة:}

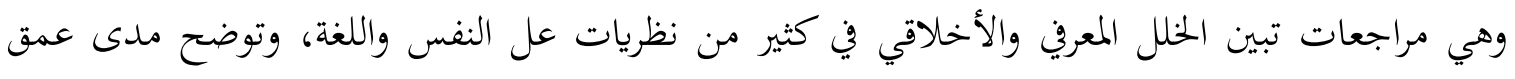
الأزمة المعرفية في الغرب. وعلم اللغة ذاته يبين مدى قصور الفكر العلماني (الموضعي)، ويذهب بعضهم إلى 
أن الفكر الفلسفي الحقيقي في الغرب يوحد الآن في الكتابات التي تتناول فلسفة اللغة، وليس في كتب الفلسفة التقليدية.

وهذا قليل من كثير. ومما له دلالته أن الفكر المضاد ليس له أي صدى في الفكر العلماني العربي الذي لا يزال يدور في إطار مفاهيم استوردوها من غرب القرنين الثامن عشر والتاسع عشر، أي أن العلمانيين بهذا المعنى ليسوا علميين بما فيه الكفاية، ولم يلحقوا بأوروبا كما يزعمون.

ويمكن حصر الأعمال التي كتبها مفكرون في العالم الثالث في الخط النقدي نفسه للحضارة الغربية للاستفادة منها. ولعل كتاب "إشكالية التحيز: رؤية معرفية ودعوة للاجتهاد"، الذي صدرت طبعته الأولى عام 1994م* محاولة أولية في هذا الاتحاه. وأعتقد أنه لا بد من وجود باحثين في الولايات المتحدة مهمتهم رصد مثل هذه الدراسات ومتابعتها وجمعها وجعلها متاحة للدارسين وللرأي العام في العالم الإسلامي.

يمكن القول إن المتتالية العلمانية والنموذج العلماني مع أنه تحقق في التاريخ إلا أنه يحوي جرثومة هلاكه داخله، ولكنه مع هذا تمكن من الاستمرار مدة طويلة لسببين:

$$
\text { أ- الإمبريالية التي حققت لشعوب الغرب مكاسب مادية ضخمة من خلال غب شعوب العالم. }
$$

ب- تحققت المتتالية العلمانية بالتدريج ولم تتحقق دفعة واحدة، فبينما تمت علمنة الاقتصاد والسياسة ظلت أخلاق الناس تتبع المنظومة المسيحية. وعلى الرغم من هذا بدأت أزمة الحضارة العلمانية الغربية في التفاقم ابتداءاً من أواخر القرن التاسع عشر، ومع تحقق المتتالية العلمانية في جميع المجالات، ومع منتصف القرن العشرين دخلت المجتمعات الغربية مرحلة الأزمة المستمرة (الأخلاقية والبيئية... الخ).

لقد أصبح الحديث عن الأزمة موضوعاً أساسياً كامناً ظاهراً في الكتابات الغربية، ويتواتر في معظم الدراسات والأعمال الفنية الغربية الحديثة. ودارس الآداب الغربية الحديثة يعرف أكثر من غيره أن فكرة الأزمة، بل الكارثة، فكرة أساسية في هذه الآداب. 
ولعل أهم الأعمال الأدبية الغربية في العصر الحديث هي قصيدة ت. س. إليوت: "الأرض الخراب" (التي يرى بعض النقاد أها "ملحمة" الإنسان الغربي الحديث)، وهي قصيدة تتحدث عن أرض أصيبت بالعقم والجدب والجفاف، تبحث دون جدوى عن مخرج، فقد فقدت حتى المقدرة على التوبة والخلاص، وقصيدة: الأرض الخراب ليست فريدة ولا شاذة، وإنما تعبر عن حقيقة أساسية في الحضارة الغربية، وهي أفها حضارة في أزمة. واصطلاح "أزمة الحضارة الغربية الحديثة" ليس من اختراعنا، وإنما هو من نحت مؤرخين ومفكرين غربيين مثل: شوبنهاور وشبنجلر وتويني وكولن وويلسون.

ونخن إذا سألنا المتخصصين -كلاًَ في تخصصه- فإننا سنجد أن موضوع الأزمة هذا له أصداؤه في معظم التخصصات. ونظراً لأن الحضارة الغربية هي أولى التجارب الإنسانية في إنشاء مجتمع علماني حديث، فإن دراسة عوامل الأزمة التي تعتمل فيه مسألة قمنا، فهي -دراسة في فاية الأمر - لما قد يحل بنا من مشاكل إن انتهجنا النهج نفسه.

ولا أعرف هل تمت دراسة منهجية لطبيعة أزمة الحضارة الغربية أم لا؟ وهل تم ربط أشكال الأزمة المختلفة الواحدة بالأخرى أم لا؟ وهل تم التوصل لآليات هذه الأزمة وأسباها ونتائجها؟ وهل درس أحد -على سبيل المثال لا الحصر - احتمال وجود علاقة ما بين مذهب المنفعة واللذة من جهة والاغتراب والإيدز) من جهة أخرى؟ (وهناك الآن من الدراسات ما يؤكد هذه المقولة. هل ثمة علاقة بين الإباحية (تبديد الجسد) وتلوث البيئة وتزين آلاف الأطنان من الأسلحة التي تكفي لتدمير العالم عشرين مرة أو تزيد (تبديد الكون)؟ هل هناك علاقة بين العقلانية والإبادة (الهولوكوست)؟ أليست الإبادة تطبيقاً منهجياً لمبدأ المنفعة على البشر، فيباد من لا نفع لهم (مثل العجزة والسلاف واليهود)، ويمنح البقاء لمن له نفع وفائدة؟ هل هناك سبيل لتحاشي الأزمة أم أن هناك "حتمية" تاريخية في أن تسير هذه الحضارة (وأن نسير نحن)، في هذا المسار، وأن تقع هذه الحضارة (ونقع نحن) في هذا المأزق (كما يصر بعض دعاة التحديث والتغريب)؟ أي: أن المطلوب التوصل إليه هو "نظرية عامة" لأزمة الحضارة الغربية الحديثة وأزمة النمط التحديثي الغربي. 
وأعتقد أنه قد حان الوقت لعقد مؤتمر يهدف لدراسة هذا الموضوع دراسة علمية تحليلية شاملة، بحيث يمكن أن ننشئ علماً أو تخصصاً نطلق عليه "علم الأزمة"، يهدف إلى الإجابة عن بعض الأسئلة التي طرحناها، وتتم فيه دراسة أزمة الحضارة الغربية من وجوهها كافة، وتُرصد الموضوعات الأساسية الكامنة لهذا الأزمة في التخصصات والنشاطات كافة، ثم يتم ربطها الواحدة بالأخرى، سعياً إلى تطوير آليات الرصد والربط حتى نصل إلى نموذج له مقدرة تحليلية وتفسيرية عالية.

وكل الموضوعات السابقة إنما تدور حول أزمة الحضارة الغربية ونموذجها العقلاني المادي، وتنطوي على دعوة لإعادة النظر فيه. وما سميناه عِلماً يضم كل التخصصات العلمية "الطبيعية والإنسانية" الممكنة، ويتميز بأنه سيشكل نقطة التقاء بين كل المدارس والاتحاهات الفكرية، فالمتحيزون للمناهج الغربية في التفكير لن يجدوا غضاضة في الإسهام في مثل هذا المؤتمر، فهو لن يقدم خطباً حماسية أو شجباً وإنما سيحاول أن يقدم رؤية متكاملة لحضارة الغرب الحديثة باستخدام أدوات التحليل العلمية المتعارف عليها، كما أن مثل هذا المؤتمر سيحاول أن يدرس رؤية الغرب ذاته لأزمته (وثم أدب ثري يتناول هذا الموضوع).

أما المدافعون عن المشروع الإسلامي المستقل فلهم مصلحة مباشرة وخاصة في مثل هذه الدراسة، لأن النموذج التحديثي الغربي هو النموذج المضاد لكل الخصوصيات "الأثنية" والدينية، وهو التطبيق العلماني لما يسمى بالعلمانية، وأزمته هي في جوهرها أزمة النموذج العلماني.

وأهم مباحث هذا العلم الجديد هو "ثثن التقدم". وأقترح أنه قد حان الوقت لأن نتسلح بالشجاعة، ونحاول أن نحول الكيف إلى كم (حتى يمكن للناس فهم ما نقول)، فنأتي لظواهر مثل الأمراض الاجتماعية المختلفة (المخدرات، الإباحية، تآكل الأسرة، الشذوذ الجنسي)، وهي ظواهر ذات طابع بنيوي في المجتمعات الغربية التي يقال لها متقدمة ونخسب تكلفتها المادية الظاهرة كما نخوِّل تكلفتها المعنوية الخافية والكامنة إلى أرقام. فمن ضمن تكاليف الطلاق مثلاً تشتت الأطفال وزيادة التخريب في المدارس، وحالات الاهيار العصبي. (ومن المعروف أن شركات التأمين في الولايات المتحدة تزيد تكاليف التأمين على المطلقين) ويمكن أن نحسب أيضاً تأكل الأسرة، وزيادة معدلات الطلاق، وازدحام الشوارع). وبالنسبة للمشروع الصناعي عموماً تحسب تكلفة التلوث على سبيل المثال وثقوب الأوزون. وثمة إحصائية تذهب إلى أنه لو حسبت التكاليف الحقيقية 
لأي مشروع صناعي لظهر أنه مشروع خاسر، وأن المشروع الصناعي الغربي قد حقق ما حقق من نجاح واستمرار لأن الآخرين دفعوا الثمن، وأنه مع نجاح بحربة بعض الدول في اللحاق بالغرب بدأت تظهر الكارثة الكونية التي تتواتر أخبارها في وسائل الإعلام يومياً.

\section{ثالثاً: دراسات نقدية: في خصوصية الحضارة الغربية}

ومثل هذا الدراسات تمدف إلى نزع صفة العالمية والعلمية والإطلاق عن الحضارة الغربية، وتبين أن كثيراً من "القوانين العلمية" التي يدافع عنها دعاة التغريب على أساس أغا تصلح لكل زمان ومكان هي نتيجة تطور تاريخي وحضاري محدد، وثمرة تضافر ظرف فريدة في لحظة فريدة. وقد تراكمت مثل هذا الدراسات الآن في العالم الغربي. كما أننا يمكنا أن نسهم فيها أيضاً بكتابة دراسات في هذا الموضوع. ومن هذا الصنف من

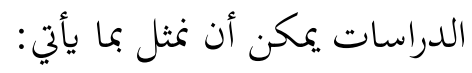

\section{أ- نظرية ماكس فيبر في عملية الترشيد بوصفها سمة أساسية للحضارة الغربية:}

تدور كتابات ماكس فيبر عالم الاجتماع الألماني الشهير حول موضوع أساسي كامن، وهو خصوصية الحضارة الغربية، وهو يرى أن الخاصية الأساسية لهذا الحضارة هي ابتاهها نحو الترشيد (المادي) والمزيد من الترشيد، إلى أن يتم ترشيد كل جوانب الحياة. وهذا الخاصية تعود كما يرى فيبر إلى سمات بنيوية فريدة مقصورة على كلى الحضارة الغربية ومكوناها الفريدة، مثل: القانون الروماني، وبناء المدينة الغربية، وبعض سمات اليهودية، ثم المسيحية الغربية، بل وتطور الموسيقى في الغرب.

وعملية الترشيد هذه في تصوره هي التي أدت إلى ظهور ما يسميه بالرأسمالية الرشيدة -في مقابل رأسمالية المجتمعات التقليدية- التي طورت نظم الإدارة البيروقراطية الدقيقة الموضوعية. وهو يرى أن عملية الترشيد هذه لن تؤدي إلى إسعاد الإنسان، وإنما إلى ظهور ما يسميه بالقفص الحديدي، أي أن يخضع البشر لقواعد عامة رشيدة صماء، إذ أن الترشيد الغربي ينصرف إلى القواعد والإجراءات دون الأهداف، فالأهداف النهائية بطبيعتها أمور مطلقة أخلاقية. ومهما كان الأمر، فإن تركيز فيبر على خصوصية الحضارة الغربية (في مقابل 
تركيز ماركس أو دوركهايم على فكرة القانون العام) أمر في غاية الأهية ويخدم قضية المشروع المعري الإسلامي المستقل.

ب- خصوصية تطور (التكنولوجيا) الغربية الحديثة:

وذلك في مقابل (التكنولوجيات) الأخرى، مثل (التكنولوجيا) الصينية على سبيل المثال، أو حتى

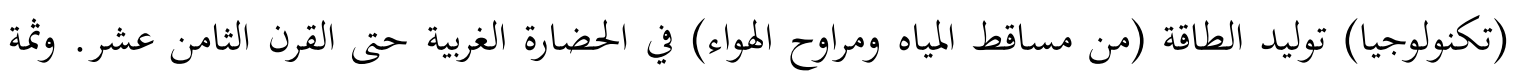
نظرية تذهب إلى أن (تكنولوجيا) توليد الطاقة المبنية على التعدين التي ظهرت في الغرب في فاية القرن الثامن

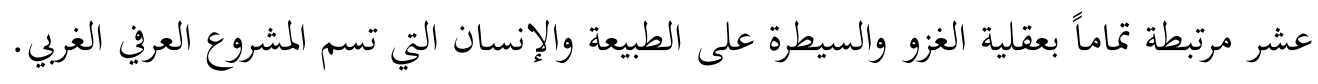

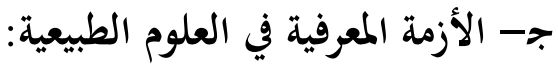

من أهم التخصصات التي عبر التفكير المضاد عن نفسه من خلالها الفكر الفلسفي في العلوم الطبيعية. فعلم

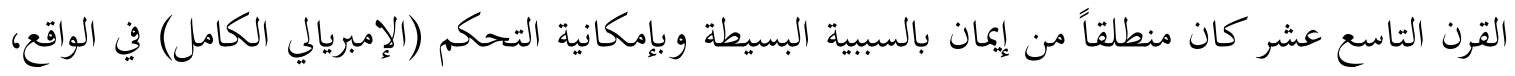
وبأن رقعة الجههول تتناقص نتيجة لتزايد رقعة المعلوم. وقد تحطمت السبيية البسيطة تماماً، ولم يعد أحد يحلم

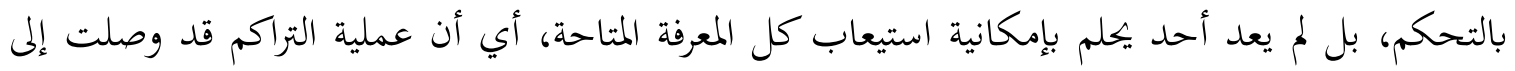

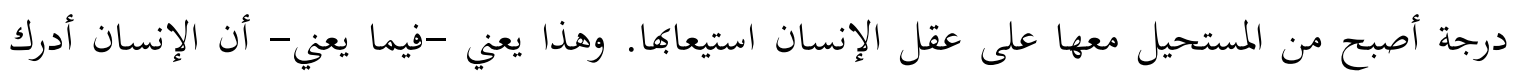
حدوده من خلال انتصاراته (وهذه مفارقة تستحق التأمل).

كما أن وهم تناقص الجمهول وتزايد المعلوم سقط تماماً، فلمعلوم يتزايد، ولكن بتزايده يتزايد المجهول، وهذه مفارقة أخرى. وقد تجلى هذا في المفاهيم والفرضيات والنظريات بل والقوانين العلمية الحديثة، وتولت العلوم الطبيعية إلى علوم إجرائية تطمح إلى تفسير بعض جوانب الظاهرة لا كل جوانبها. ولا بد من دراسة هذا الموضوع الذي يجب أن يعطى أولوية حتى يتحرر المتعلمون والمثقفون من وهم السبيية البسيطة ومن النماذج المانج التراكمية التي تضفي على الغرب صيغة الإطلاق والمركزية. 
في محاولتنا أن نستعيد للغرب نسبيته، وأن ننزع عنه إطلاقه ومركزيته، تشكل اليابان نموذجاً آخر مهماً. فهو نموذج تنموي مادي شبه روحي، ولكنه اختط طريقاً حضارياً غير الطريق الغربي، كما أنه احتفظ بكثير من العناصر التراثية بل ووظفها في عملية التحديث والتطوير. ويمكن هنا أيضاً الاستفادة من أدبيات التفكير الاحتجاجي الغربي، فقد ظهرت تواريخ عديدة لحضارة الإنسان من منظور عالمي حق، وليس من منظور متمركز حول الغرب.

ويمكن في هذا المضمار دراسة تواريخ الشعوب الإسلامية غير العربية بكل ما تحوي من ثراء وتنوع، ينتظمها مع هذا إطار إسلامي واحد، بحيث يصبح هذا في حد ذاته دليلاً على إمكانية إنشاء حضارة تعددية حقيقية لا تسقط بالضرورة في العدمية النسبية التي تؤدي إليها التعددية الغربية.

ه- دراسة الأسس الدينية والثقافية للعلم الغربي وللمفاهيم الغربية:

ظهرت دراسات عديدة تبين علاقة الرؤية المسيحية (الغربية) للتاريخ بمفاهيم مثل التقدم. بل إني قرأت إحدى الدراسات تسمى: ماركس والبعث يذهب مؤلفها إلى حدود معادلتين متوازيتين متساويتين:

الأولى: ميلاد المسيح، وصلب المسيح، وبعث المسيح. والثانية ظهور الإنسانية الشيوعية البدائية، والصراع الطبقي، وثورة (البروليتاريا) والحكومة الشيوعية. كما تشير هذه الدراسة إلى أن هناك دراسات مشابكة عن

$$
\text { أصول العلوم والفنون الغربية. }
$$

\section{و- بعض المصادر اليهودية للفكر الغربي الحديث:}

يطرح الفكر الغربي الحديث نفسه على أنه فكر عالي، وهكذا يروج له دعاة الحداثة. ولكن من المتفق عليه بين دراسي هذا الفكر أن اليهود الغربيين قد حققوا بروزاً غير عادي داخل الحضارة الغربية الحديثة. ولكن لم تؤكد الدراسات المنشورة ما يمكن أن نسميه المكون "اليهودي" في الفكر الغربي الحديث. وكلمة "يهودي" هنا لا تعني يهودية التوراة أو حتى التلمود، وإنما هي يهودية القبالة (خاصة القبالة اللوريانية) التي سيطرت تماماً على اليهود وعلى المؤسسات الدينية اليهودية ابتداءاً من القرن السادس عشر . 
والقبالة اللوريانية باختصار شديد: هي فلسفة حلولية متطرفة، توحد تماماً بين الخالق والمخلوق حتى تتلاشى الفوارق بينهما، وحتى يصبح الإنسان هو الخالق، وتصبح الطبيعة هي أيضاً الخالق. ومن ثم نجد أن القبالة اللوريانية تعد تمهيداً لفكر العلماني الذي يقدس المادة والطبيعة والإنسان، ليس بوصفه مخلوقاً فريداً، وإنما بوصفه جزءاً من الطبيعة. والقبالة اللوريانية لها تصورها ورؤيتها للكون ولدوراته، وللتاريخ ومساره، ولإنسان وحركاته وسكناته. وقد أدت إلى ما يسمى في الدراسات اليهودية بتصاعد الحمى الماشيحانية (أي الاعتقاد بقرب وصول المسيح المخلص اليهودي). والنزعة الماشيحانية نزعة عدمية معادية للحدود، ترى الإنسان كائناً لا حدود له (أي أنه إله)، ولذا فالنزعة الماشيحانية تترجم نفسها دائماً إلى ابحاهات ترخيصية إباحية تسقط

$$
\text { الشرائع الإلهية والإنسانية. }
$$

ولم تظهر دراسة واحدة باللغة العربية عن القبالة اللوريانية، أما الحركات الماشيحانية فقد ظهر كتاب أو أكثر يتحدث عنها دون دراسة للأنماط الكامنة أو الدلالات الفلسفية والتاريخية المضمرة فيها. وعدد الدارسين للقبالة اللوريانية والحركات الماشيحانية اليهودية في الغرب محدود، كما أفم محصورون داخل حقل الدراسات اليهودية لا علاقة لهم بالحركات الفكرية الغربية الحديثة.

ومؤرخو الفكر الغربي الحديث لا يعرفون الكثير عن القبالة اللوريانية أو لعلهم يجيهلوها تماما. ومن ثم ظل المكون اليهودي (القبالي) للفكر الغربي الحديث خفياً، ولم يتوافر أحد على دراسته بشكل منهجي مستفيض، وهذا ما قد يمكنا إنجازه، أو على الأقل وضع الخطوط الأساسية لإنجازه. ويمكن أن يتم هذا على

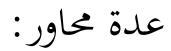

الغنوصية: من الموضوعات المتواترة الآن في الكتابات الغربية الفكر الغنوصي ومدى ثأثيره في النظرية السياسية الغربية الحديثة. وفي تصوري أن القناة الأساسية للغنوصية هي القبالة اللوريانية وكتابات المفكرين اليهود المتأثرين بها. ويلاحظ وجود نزعة غنوصية قوية في كتابات بعض المفكرين الغربيين من اليهود. ودراسة الغنوصية أمر مهم في حد ذاته، إذ لا يمكن فهم كثير من الحركات الباطنية (غلاة الشيعة، الزنادقة) والحركات السرية ذات الاتجاهات العلمانية (الفلسفة الربوبية، الماسونية) إلا بدراسة الغنوصية. 
باروخ سبينوزا: يعد سبينوزا في تصور الكثير من مؤرخي الفكر الغربي الحديث فيلسوف العلمانية الأول، بل ويككن رؤيته على أنه أول مفكر علماني، فقد ترك اليهودية ولم يعتنق دينا آخر. ومن المهم للغاية توضيح علاقة فكره بالفكر القبالي (والغنوصي). وهناك دراسات تشير إلى هذا الموضوع دون التعمق فيه.

سيجموند فرويد: لا يككن فهم مدرسة التحليل النفسي برموزها الجنسية الكثيرة إلا بالعودة للقبالة اللوريانية التي وصفت بأخها "جنست الإله وألمت الجنس". وقد ظهر العديد من الدراسات في هذا الموضوع. بل هناك دورية متخصصة عن علاقة اليهودية بالتحليل النفسي. وقد قمت بتجميع بعض المواد في هذا الموضوع، وإن كان الأمر يحتاج لجهد أكثر منهجية وتكثيفاً.

فرانز كافكا: يعد كافكا من أهم الروائين الغربيين، ويعد موقفه العبثي العدمي تعبيراً عن "أزمة الإنسان في العصر الحديث" (أي أزمة الإنسان الغربي في العصر الحديث). والمكون اليهودي القبالي في فن كافكا وفكره

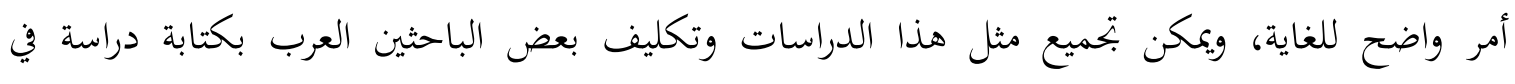
الموضوع، وترجمة بعض الدراسات الغربية.

المدرسة التفكيكية: (ما بعد المحاثة): (

تعد المدرسة التفكيكية أهم المدارس الفكرية الغربية في الوقت الحاضر. وقد أسس هذه المدرسة "دريدا"

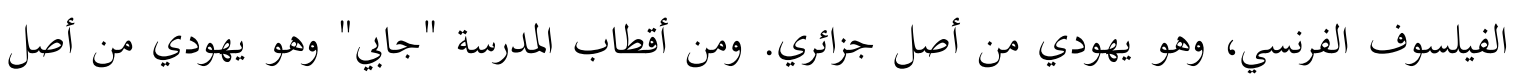

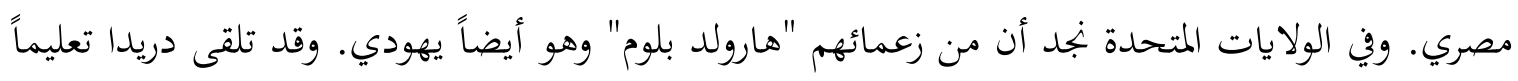

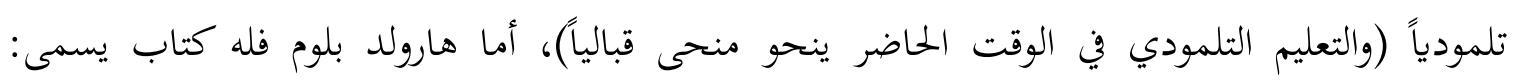

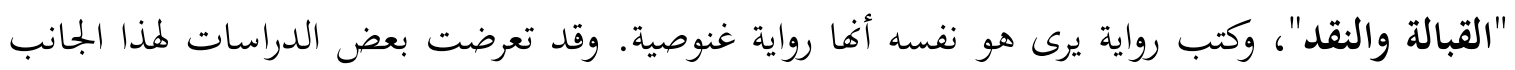

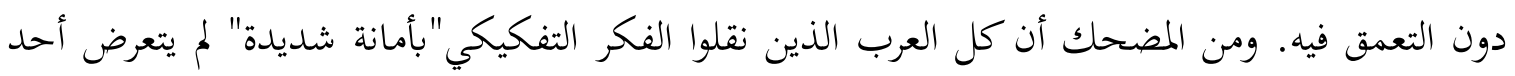

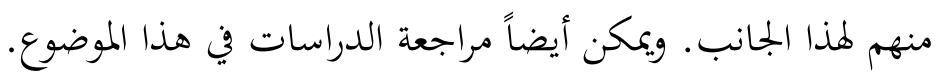

رابعاً: الإمبريالية بوصفها مقولة تحليلية خامساً: العلمانية الرأسمالية والاشتراكية 
قدمنا في موسوعة اليهود واليهودية والصهيونية مخططاً مبدئياً ليكون خطة عمل لمزيد من الدراسات، ويمكن أن يصبح الجزء النظري فيها ورقة عمل مبدئية في هذا المجال. 